\title{
Synthesis of $N$-Z, $N^{\prime}$-Formyl $\alpha$-Amino Acid Derived Gem-Diamines
}

\author{
Vommina V. Sureshbabu • N. Narendra
}

Accepted: 28 March 2008/Published online: 29 April 2008

(C) Springer Science+Business Media, LLC 2008

\begin{abstract}
A variety of $N$-carbobenzoxy, $N^{\prime}$-formyl gemdiaminoalkyl derivatives have been obtained through Goldsmith-Wick reaction of $\mathrm{Z}$ - $\alpha$-amino acid/peptide acid derived isocyanates with $96 \% \mathrm{HCOOH}$ in presence of 4-dimethylaminopyridine (DMAP) as catalyst. The reaction proceeds to completion within $2-4 \mathrm{~h}$ and results in good yields of the products isolated as stable solids.
\end{abstract}

Keywords Z- $\alpha$-amino acids - Acyl azides - Isocyanates · DMAP · Goldsmith-Wick reaction · Formylation · Gem-diamines

\section{Introduction}

Formamides are medicinally and synthetically useful class of compounds. They form precursors for the preparation of wide range of important organic compounds like formamidines, monomethylated amines, nitrogen containing hetrocycles and in Vielsmier formylation reactions. As catalysts, they are employed in asymmetric allylations and hydrosilylation of carbonyl compounds. $N$-Formyl amino acid esters are employed in enzymatic synthesis of peptides in aqueous media. The formyl group also serves as an effective $N$ as well as $O$ protecting group (Sarvari and Sharghi 2006). They are dehydrated to isonitriles which form starting materials for 1-substituted tetrazoles, oxazolines, pyrroles and are the key components in Passerini and

V. V. Sureshbabu $(\varangle) \cdot$ N. Narendra

Peptide Research Laboratory, Department of Studies in Chemistry, Central College Campus, Bangalore University, Dr. B. R. Ambedkar Veedhi, Bangalore 560001 ,

Karnataka, India

e-mail: hariccb@rediffmail.com
Ugi's multi component reactions (Domling and Ugi 2000). Synthesis of partially modified retroinverso peptides (PMRIs) using $N$-formyl protected amino acids has also been explored (Chorev and Goodman 1983; Chorev 2005).

The conversion of amino group of amino acid ester into formamide results in the $N$-formylated amino acid derivative as shown in the Fig. 1. A large number of efficient protocols have been reported for achieving this type of transformation. On the other hand, $N$-formamide group can also be inserted in the place of $\alpha$-carboxyl group of $N$-protected amino acid. Since formamide group is the derivative of amine, a synthesis of this kind can be visualized possible in view of the availability of methods for converting the $-\mathrm{COOH}$ group into amine through Curtius or Hoffmann rearrangement (Fletcher and Campbell 1998). Derivatization of the newly obtained amine with formic acid leads to functionalized formamides (Fig. 2), a useful class of synthons. They are employable as starting materials to access synthetically valuable $N$-urethane protected compounds, for instance, urethane protected $\alpha$-amino isonitriles.

The reported protocols for converting the $\alpha$-amino group into formamide moiety include reacting the amines directly with formic acid or in presence of a catalyst like $\mathrm{ZnO}$ and formylating agents like $\mathrm{KF}-\mathrm{Al}_{2} \mathrm{O}_{3}$, chloral, acetic formic anhydride, activated formic acid using DCC/EDC, formic acid esters, ammonium formates, 2-chloro-4,6-dimethoxy[1,3,5] triazole (CDMT), cyanomethyl formate and supported reagents (Sarvari and Sharghi 2006).

Nevertheless, there were no reports in the literature (Scheibler and Chorev 2003) concerning to the conversion of carboxyl group of $N$-protected amino acid into the corresponding formamide moiety until our group recently published a note on the synthesis of $N$-Fmoc, $N^{\prime}$-formyl gem-diaminoalkyl derivatives from Fmoc amino acids 
Fig. $1 N$-Formyl amino acid ester
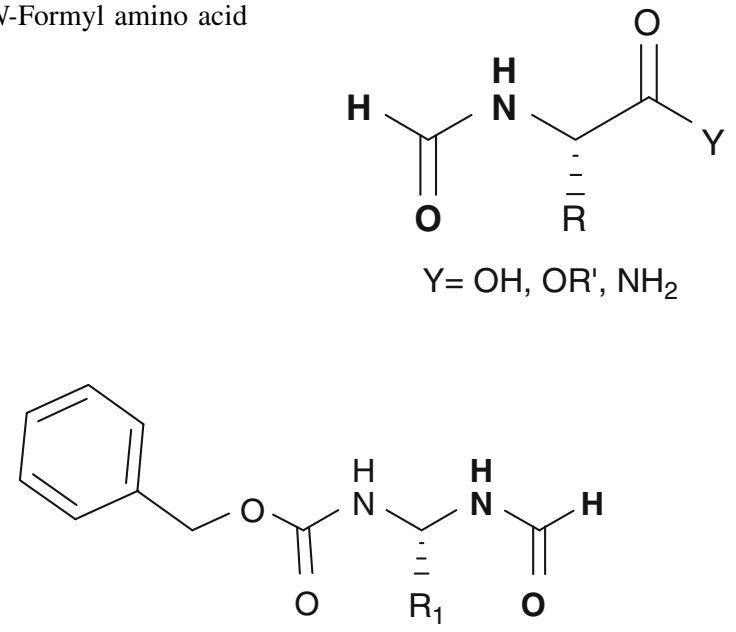

Fig. $2 N$-Benzyloxycarbonyl, $N^{\prime}$-formyl gem-diamine

(Sudarshan et al. 2007). The synthesis of such compounds would require generation of a gem-diamine (Fuller et al. 1985) precursor through any of the known methods (Fletcher and Campbell 1998) and then reacting with suitable formylating agent. But since the alkyl gem-diamines are known to decompose into amides, aldehydes and amines (Loudon et al. 1981; Parham and Loudon 1978) and are difficult to prepare in good yields, an alternate route devoid of the generation of gem diamine is required.

The synthesis of the related $N$-formylated gem diamines particularly useful as intermediates for PMRIs has been reported (Chorev and Goodman 1983; Berman and Goodman 1984). This synthesis employs $N$-formyl protected $\alpha$-amino acids as starting materials and converts them into corresponding acyl azides followed by the rearrangement of the latter into isocyanates. The $N$-formyl $\alpha$-amino-isocyanates are then trapped with benzyl alcohol or tert-butyl alcohol to yield $N$-formyl, $N^{\prime}$-benzyloxycarbonyl or tertbutyloxycarbonyl gem diamines. But in our hands, coupling of alcohols to isocyanates was slow and required long hours of reflux or microwave acceleration (Venkataramanarao et al. 2007). In contrast, formylation of isocyanates via Goldsmith-Wick reaction was recently reported to take place rapidly in the presence of 4-dimethylaminopyridine
(DMAP) and at $0^{\circ} \mathrm{C}$ and offers practically attractive route to access the title compounds (Schuemacher and Hoffmann 2001). However, Goodman and co-workers have thoroughly studied the usage of urethane protected aminoisocyanates as synthetic intermediates and have shown that they are prone to multiple side reactions essentially arising from the heterolytic cleavage of C-NCO bond in polar solvent (Chorev 1984). Pallai and co-workers have reported similar results on the instability of urethane protected gem-diamines obtained from the oxidative rearrangement of corresponding amides (Pallai 1983). Despite this, we envisioned that unlike the alcoholysis of the isocyanates under high temperature reflux and highly polar methanolic reaction medium, as described by Goodman et al., Goldsmith-Wick formylation proceeds under very mild reaction conditions and in non-polar $\mathrm{CH}_{2} \mathrm{Cl}_{2}$ solvent and hence would minimize the likely side reactions of urethane protected aminoisocyanates. Also in recent years, the synthetic utility of $N$-Fmoc/Boc/Z-protected isocyanates is being explored with respect to the preparation of peptidomimetics.

In this context, we proceeded with the direct reaction of $\mathrm{Z}$ - $\alpha$-amino-isocyanates with formic acid. $\mathrm{Z}$ group was selected in view of its practical utility for solution phase peptide synthesis when compared with Fmoc-chemistry. It enables the deprotection employing catalytic hydrogenation under neutral conditions thus circumventing the acid catalyzed degradation of gem-diamines. We report in this paper an efficient synthesis of $N$-formyl, $N^{\prime}$-benzyloxycarbonyl gem-diamines by the reaction of $\mathrm{Z}$ - $\alpha$-amino isocyanates with formic acid (Scheme 1).

The synthetic utilities of $N$-urethane protected $\alpha$-aminoisocyanates in the preparation of peptidomimetics have been documented in the literature. Isocyanates derived from azides of $N$ protected amino acids and peptide acids have been employed in synthesis of peptidomimetics like peptidyl ureas (Patil et al. 2003; Sureshbabu et al. 2006) and PMRIs. Fischer et al., recently reported the conversion of a series of $\mathrm{Z}$ - $\alpha$-amino isocyanates into active $N$-hydroxy succnimidyl carbamates employed as building blocks to assemble ureidodipeptides (Fischer et al. 2007). Venkataramanarao and Sureshbabu have described the synthesis of Fmoc protected
Scheme 1 Synthesis of $\mathrm{N}$-formylated gem-diamines from Z-amino acids

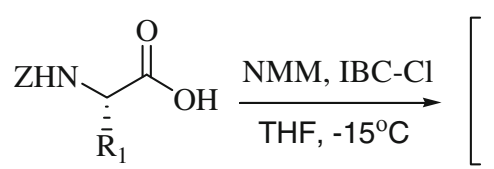

1a-l

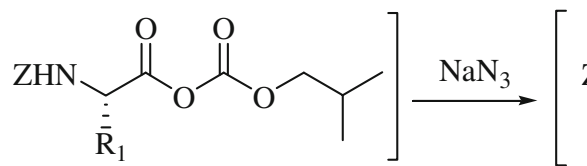

2a-1<smiles>[Z1]NC([R])C(N)=O</smiles>

3a-l

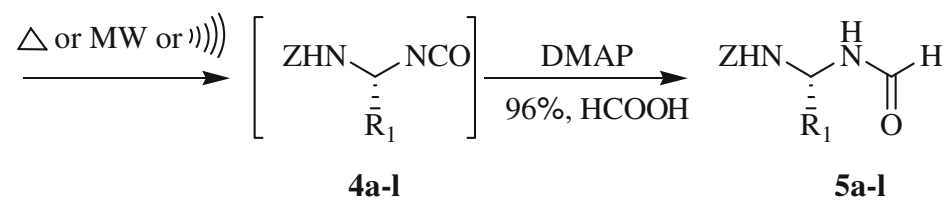


PMRIs by reacting the isocyanates derived from Fmocamino acids with Boc/Z- amino acids in presence of DMAP through Goldsmith-Wick reaction (Venkataramanarao and Sureshbabu 2006). Herein we present a novel and useful application of $\mathrm{Z}$ - $\alpha$-amino acid derived isocyanates through the synthesis of the title functionalized formamides.

\section{Materials and Methods}

The amino acids were obtained from Sigma-Aldrich Company. Melting points were determined using capillary method and are uncorrected. LG domestic microwave oven (LG MS 194A) operating at $2,450 \mathrm{MHz}$ was used for the preparation of isocyanates. Ultrasonication under TRANSSONIC T 310/H Ultrasonic bath, IR spectra were recorded on a Nicolet model impact 400D FT-IR spectrometer $(\mathrm{KBr}$ pellets, $3 \mathrm{~cm}^{-1}$ resolution), ${ }^{1} \mathrm{H}$ NMR spectra were recorded on a Bruker $400 \mathrm{MHz}$. Mass spectra were recorded on ESMS (HP 1100 series, MSD single quadrapole). The TLC was effected with silica gel GF254 obtained from MERCK Chemicals on pre-coated glass plates using as mobile phases the following solvent systems: (a) chloroform:methanol (9:1) and (b) ethyl acetate:hexane (6:4). All solvents were freshly distilled prior to use. The $N^{\alpha}$-Z-dipeptide acids were prepared via mixed anhydride method by employing $O, N$-bis-trimethylsilyl amino acids using the reported procedures (Tantry and Sureshbabu 2004). All the six Z-peptides made were purified and characterized prior to use.

Characterization data of Z-dipeptide acids

Z-Ala-Val-OH: M.p $97^{\circ} \mathrm{C} ; \quad{ }^{1} \mathrm{HNMR} \quad\left(\mathrm{CDCl}_{3}\right):-1.10$ $(\mathrm{d}, 6 \mathrm{H}), 1.51(\mathrm{~d}, 3 \mathrm{H}), 2.62(\mathrm{~m}, 1 \mathrm{H}), 4.59(\mathrm{~m}, 1 \mathrm{H}), 4.73$ (m, 1H), $5.42(\mathrm{~s}, 2 \mathrm{H}), 6.58(\mathrm{~m}, 1 \mathrm{H}), 7.12-7.25(\mathrm{~m}, 5 \mathrm{H})$, 7.53 (m, 1H); ${ }^{13} \mathrm{CNMR}:-17.8,18.1,35.3,52.3,60.5$, 68.2, 128.5, 129.2, 131.0, 149.3, 154.9, 175.9, 178.9 .

Z-Leu-Val-OH: M.p $82{ }^{\circ} \mathrm{C}$; ${ }^{1} \mathrm{HNMR}\left(\mathrm{CDCl}_{3}\right)$ :- 0.94-1.10 (m, 12H), $1.81(\mathrm{~m}, 1 \mathrm{H}), 1.91(\mathrm{~m}, 1 \mathrm{H}), 2.17(\mathrm{~m}, 1 \mathrm{H}), 4.39$ (m, 1H), $4.42(\mathrm{~m}, 1 \mathrm{H}), 5.40(\mathrm{~s}, 2 \mathrm{H}), 6.61(\mathrm{~m}, 1 \mathrm{H}), 7.12-$ 7.25 (m, 5H), 7.49 (m, 1H); ${ }^{13}$ CNMR:- 17.6, 21.3, 22.9, $34.8,41.8,52.3,61.1,69.0,128.3,128.9,131.5,148.1$, 155.2, 175.3, 178.9 .

Z-Phe-Ala-OH: M.p $158^{\circ} \mathrm{C} ;{ }^{1} \mathrm{HNMR}\left(\mathrm{CDCl}_{3}\right)$ :- 1.31 (d, 3H), 2.8-3.2 (m, 2H), $4.81(\mathrm{~m}, 1 \mathrm{H}), 5.12(\mathrm{~m}, 1 \mathrm{H}), 5.30$ (s, 2H), $6.41(\mathrm{~m}, 1 \mathrm{H}), 7.10-7.36(\mathrm{~m}, 10 \mathrm{H}), 7.51(\mathrm{~m}, 1 \mathrm{H})$; ${ }^{13}$ CNMR:- 17.0, 36.2, 52.5, 60.8, 68.8, 125.3, 126.1, 126.8, 128.3, 129.1, 130.9, 139.8, 149.1, 155.0,175.1, 178.0.

Z-L-Phg-Ala-OH: M.p168 ${ }^{\circ} \mathrm{C} ;{ }^{1} \mathrm{HNMR}\left(\mathrm{CDCl}_{3}\right)$ :- 1.29 $(\mathrm{d}, 3 \mathrm{H}), 4.71(\mathrm{~m}, 1 \mathrm{H}), 5.29(\mathrm{~s}, 2 \mathrm{H}), 5.91(\mathrm{~s}, 1 \mathrm{H}), 6.68(\mathrm{~m}$, 1H), 7.10-7.40 (m, 10H), 7.48 (m, 1H); ${ }^{13}$ CNMR:- 17.1, $52.9,60.8,68.3,127.1,128.3,128.9,129.2,129.8$, $131.5,137.1,149.8,155.8,175.8,178.5$.
Z-D-Phg-Ala-OH: M.p $114^{\circ} \mathrm{C} ;{ }^{1} \mathrm{HNMR}\left(\mathrm{CDCl}_{3}\right)$ :- 1.32 $(\mathrm{d}, 3 \mathrm{H}), 4.71(\mathrm{~m}, 1 \mathrm{H}), 5.29(\mathrm{~s}, 2 \mathrm{H}), 5.91(\mathrm{~s}, 1 \mathrm{H}), 6.68(\mathrm{~m}$, 1H), 7.10-7.40 (m, 10H), $7.48(\mathrm{~m}, 1 \mathrm{H}) ;{ }^{13} \mathrm{CNMR}:-17.3$, $53.0,60.8,68.1,127.2,128.5,129.1,129.3,129.8$, $131.2,137.0,150.0,155.8,175.8,178.6$.

Z-Pro-Val-OH: M.p $130{ }^{\circ} \mathrm{C} ;{ }^{1} \mathrm{HNMR}\left(\mathrm{CDCl}_{3}\right)$ :- $1.12(\mathrm{~d}$, $6 \mathrm{H}), 1.29-1.52(\mathrm{~m}, 4 \mathrm{H}), 2.68(\mathrm{~m}, 1 \mathrm{H}), 3.41(\mathrm{~m}, 2 \mathrm{H})$, $4.35(\mathrm{~m}, 1 \mathrm{H}), 4.56(\mathrm{~m}, 1 \mathrm{H}), 5.38(\mathrm{~s}, 2 \mathrm{H}), 6.78(\mathrm{~m}, 1 \mathrm{H})$, 7.15-7.27 (m, 5H), 7.51 (m, 1H); ${ }^{13} \mathrm{CNMR}:-17.4,22.9$, $28.9,30.9,47.3,52.6,61.0,69.2,128.0,128.3,130.9$, $148.7,155.9,175.2,178.8$.

Typical procedure for the preparation of $N$-Benzyloxycarbonyl, $N^{\prime}$-formyl-alkane-1,1-diamines

To a stirred solution of Z-amino acid/peptide acid $(10 \mathrm{mmol})$ in dry $\mathrm{THF}$ at $-10^{\circ} \mathrm{C}$, was added NMM $(1.1 \mathrm{ml}, 10 \mathrm{mmol})$ and ethyl chloroformate $(0.91 \mathrm{ml}$, $10 \mathrm{mmol}$ ) and stirring continued for another $20 \mathrm{~min}$ at the same temperature, then sodium azide $(0.96 \mathrm{~g}, 15 \mathrm{mmol})$ in water $(0.5 \mathrm{ml})$ was added and stirred at $0^{\circ} \mathrm{C}$ for $20 \mathrm{~min}$. The reaction mixture was evaporated at room temperature under reduced pressure. The residue was diluted with dichloromethane, washed with $10 \% \mathrm{NaHCO}_{3}(10 \mathrm{ml}), 10 \%$ citric acid $(10 \mathrm{ml})$ and water $(10 \mathrm{ml} \times 2)$. The organic layer was dried over sodium sulphate and concentrated to yield respective azides.

Further, the azide was dissolved in minimum quantity of toluene $(5 \mathrm{ml})$ and exposed to microwave irradiation at $60 \%$ total power output of the instrument for one minute or subjected to ultrasonication at room temperature for 15 $20 \mathrm{~min}$ or refluxed for $30 \mathrm{~min}$ to obtain the isocyanate. After the removal of excess toluene, the residue was diluted with dry DCM and cooled to $0^{\circ} \mathrm{C}$. To this, DMAP $(0.12 \mathrm{~g}, 1.0 \mathrm{mmol})$ was added followed by $96 \% \mathrm{HCOOH}$ $(0.57 \mathrm{ml}, 15 \mathrm{mmol})$ and stirring continued at the same temperature till the completion of the reaction as evident by precipitation of the product within $2-4 \mathrm{~h}$. The reaction mixture was diluted with $15 \mathrm{ml}$ each of water and hexane and the precipitated solid was filtered, washed with ether, $10 \% \mathrm{NaHCO}_{3}, 10 \%$ citric acid and water. The compound was dried under suction and was recrystallized using dichloromethane: hexane to obtain the product.

Characterization Data for $N$-Benzyloxycarbonyl-

$N^{\prime}$-Formylalkane-1,1-Diamines 5a-k

1. $\mathrm{N}$-Benzyloxycarbonyl- $\mathrm{N}^{\prime}$-formylmethanediamine (Z-gGly-For, 5a): IR (KBr): 1,655 and $1,710 \mathrm{~cm}^{1} ; \mathrm{R}_{\mathrm{f}}$ $\left(10 \% \mathrm{MeOH} / \mathrm{CHCl}_{3}\right): 0.30 ;{ }^{1} \mathrm{H}$ NMR $(\delta, \mathrm{DMSO})$ : $4.26(\mathrm{~m}, 2 \mathrm{H}), 4.99(\mathrm{~s}, 2 \mathrm{H}), 7.28(\mathrm{~m}, 5 \mathrm{H}), 7.96(\mathrm{~s}, 1 \mathrm{H})$, $8.03(\mathrm{~m}, 1 \mathrm{H}) ;{ }^{13} \mathrm{C}$ NMR $(\delta$, DMSO): $56.53,64.10$, $127.58,127.70,128.24,136.36,159.80,165.49$. 
2. (S)-N-Benzyloxycarbonyl- $N^{\prime}$-formylethane-1, 1-diamine (Z-gAla-For, 5b): IR (KBr): 1,658 and $1,712 \mathrm{~cm}^{1} ; \mathrm{R}_{\mathrm{f}}\left(10 \% \mathrm{MeOH} / \mathrm{CHCl}_{3}\right): 0.32 ;{ }^{1} \mathrm{H} \mathrm{NMR}$ $(\delta$, DMSO): $1.15(\mathrm{~d}, 3 \mathrm{H}, \mathrm{J}=6.8 \mathrm{~Hz}), 5.01(\mathrm{~s}, 2 \mathrm{H})$, $5.19(\mathrm{~m}, 1 \mathrm{H}), 7.27(\mathrm{~m}, 5 \mathrm{H}) 7.96(\mathrm{~s}, 1 \mathrm{H}), 8.05(\mathrm{~m}$, $1 \mathrm{H}), 8.32(\mathrm{~m}, 1 \mathrm{H}) ;{ }^{13} \mathrm{C}$ NMR $(\delta$, DMSO$): 20.83$, 56.48, 63.40, 127.71, 128.40, 137.40, 159.90, 164.12.

3. (S)-N-Benzyloxycarbonyl- $N^{\prime}$-formyl-2-phenylethane-1,1-diamine (Z-gPhe-For, 5c): IR ( $\mathrm{KBr}$ ): 1,658 and $1,705 \mathrm{~cm}^{1} ; \mathrm{R}_{\mathrm{f}}\left(10 \% \mathrm{MeOH} / \mathrm{CHCl}_{3}\right)$ : $0.30 ;{ }^{1} \mathrm{H} \quad \mathrm{NMR} \quad(\delta, \quad$ DMSO $): 2.91 \quad(\mathrm{~d}, \quad 2 \mathrm{H}$, $\mathrm{J}=7.2 \mathrm{~Hz}) 4.98(\mathrm{~s}, 2 \mathrm{H}), 5.61(\mathrm{~m}, 1 \mathrm{H}), 7.01-7.52$ $(\mathrm{m}, 10 \mathrm{H}) 7.95(\mathrm{~s}, 1 \mathrm{H}), 8.01(\mathrm{~m}, 1 \mathrm{H}), 8.21(\mathrm{~m}, 1 \mathrm{H})$; ${ }^{13} \mathrm{C}$ NMR ( $\delta$, DMSO): $37.41,54.61,66.64,127.12$, $127.18,127.81,128.24,128.41,131.56,137.40$, 159.70, 164.02.

4. (S)-N-Benzyloxycarbonyl- $N$ '-formyl-2-methylpropane-1,1-diamine (Z-gVal-For, 5d): IR (KBr): 1,660 and $1,710 \mathrm{~cm}^{1} ; \mathrm{R}_{\mathrm{f}}\left(10 \% \mathrm{MeOH} / \mathrm{CHCl}_{3}\right): 0.32 ;{ }^{1} \mathrm{H}$ NMR ( $\delta$, DMSO): $0.96(\mathrm{~d}, 6 \mathrm{H}, \mathrm{J}=7.1 \mathrm{~Hz}), 1.91(\mathrm{~m}$, $1 \mathrm{H}), 5.00(\mathrm{~s}, 2 \mathrm{H}), 5.21(\mathrm{~m}, 1 \mathrm{H}), 7.26(\mathrm{~m}, 5 \mathrm{H}), 8.11(\mathrm{br}$, $1 \mathrm{H}), 7.96(\mathrm{~s}, 1 \mathrm{H}), 8.11(\mathrm{~m}, 1 \mathrm{H}), 8.18(\mathrm{~m}, 1 \mathrm{H}) ;{ }^{13} \mathrm{C}$ NMR ( $\delta$, DMSO): 18.40, 31.72, 61.62, 65.68, 127.70, 128.40, 136.40, 155.36, 164.12.

5. (S)- $N$-Benzyloxycarbonyl- $N$ '-formyl-3-methylbutane-1,1-diamine (Z-gLeu-For, 5e): IR ( $\mathrm{KBr}$ ): 1,659 and $1,705 \mathrm{~cm}^{1} ; \mathrm{R}_{\mathrm{f}}\left(10 \% \mathrm{MeOH} / \mathrm{CHCl}_{3}\right)$ : $0.31 ; \quad{ }^{1} \mathrm{H} \quad \mathrm{NMR} \quad(\delta, \quad$ DMSO $): 0.95 \quad(\mathrm{~d}, \quad 6 \mathrm{H}$, $\mathrm{J}=5.9 \mathrm{~Hz}), 1.51-1.82(\mathrm{~m}, 3 \mathrm{H}), 4.95(\mathrm{~s}, 2 \mathrm{H}), 5.05$ $(\mathrm{m}, 1 \mathrm{H}), 7.27(\mathrm{~m}, 5 \mathrm{H}), 7.96(\mathrm{~s}, 1 \mathrm{H}), 8.12(\mathrm{br}, 1 \mathrm{H})$, $8.23(\mathrm{~m}, 1 \mathrm{H}) ;{ }^{13} \mathrm{C}$ NMR ( $\delta$, DMSO): $25.31,35.60$, 57.21, 66.68, 127.69, 128.36, 137.34, 155.12, 164.22.

6. (S)- $N$-Benzyloxycarbonyl- $N^{\prime}$-formyl-2-methylbutane-1,1-diamine (Z-gIle-For, 5f): IR (KBr): 1,655 and $1,708 \mathrm{~cm}^{1} ; \mathrm{R}_{\mathrm{f}}\left(10 \% \mathrm{MeOH} / \mathrm{CHCl}_{3}\right): 0.30 ;{ }^{1} \mathrm{H}$ NMR ( $\delta$, DMSO): $0.82(\mathrm{~m}, 6 \mathrm{H}), 1.01-1.85(\mathrm{~m}, 3 \mathrm{H})$, $4.99(\mathrm{~s}, 2 \mathrm{H}), 5.16(\mathrm{~m}, 1 \mathrm{H}), 7.26(\mathrm{~m}, 5 \mathrm{H}), 7.92(\mathrm{~s}, 1 \mathrm{H})$, $8.10(\mathrm{br}, 1 \mathrm{H}), 8.21(\mathrm{br}, 1 \mathrm{H}) ;{ }^{13} \mathrm{C}$ NMR $(\delta, \mathrm{DMSO})$ : $10.98,14.38,24.78,38.89,59.22,65.41,127.71$, 128.34, 137.20, 160.51, 164.66.

7. (S)- $N$-Benzyloxycarbonyl- $\boldsymbol{N}^{\prime}$-formyl-phenylmethane-1,1-diamine (Z-L-gPhg-For, 5h): IR ( $\mathrm{KBr}$ ): 1,662 and $1,710 \mathrm{~cm}^{1} ; \mathrm{R}_{\mathrm{f}}\left(10 \% \mathrm{MeOH} / \mathrm{CHCl}_{3}\right): 0.29$; ${ }^{1} \mathrm{H}$ NMR ( $\delta$, DMSO): $5.00(\mathrm{~s}, 2 \mathrm{H}), 6.20(\mathrm{~m}, 1 \mathrm{H})$, 7.10-7.45 (m, 10H), $7.93(\mathrm{~s}, 1 \mathrm{H}), 8.11(\mathrm{~m}, 1 \mathrm{H}), 8.20$ (br, $1 \mathrm{H}) ;{ }^{13} \mathrm{C}$ NMR ( $\delta$, DMSO): 59.59, 69.11, 123.95, $125.30,127.71,128.34,128.96,135.01,137.15$, 159.32, 162.21.

8. (R)- $N$-Benzyloxycarbonyl- $N^{\prime}$-formyl-phenylmethane-1,1-diamine (Z-D-gPhg-For, 5i): IR ( $\mathrm{KBr}$ ): 1,657 and $1,710 \mathrm{~cm}^{1} ; \mathrm{R}_{\mathrm{f}}\left(10 \% \mathrm{MeOH} / \mathrm{CHCl}_{3}\right)$ : $0.29,{ }^{1} \mathrm{H}$ NMR $(\delta$, DMSO): $5.01(\mathrm{~s}, 2 \mathrm{H}), 6.22$ (m, 1H), 7.10-7.50 (m, 10H), $7.95(\mathrm{~s}, 1 \mathrm{H}), 8.10(\mathrm{~m}$, $1 \mathrm{H}), 8.22(\mathrm{br}, 1 \mathrm{H}) ;{ }^{13} \mathrm{C}$ NMR ( $\delta$, DMSO): 59.61, $69.15,123.94,125.32,127.73,128.35,128.96$, $135.05,137.15,159.31,162.29$.

9. (S)- $N$-Benzyloxycarbonyl- $N$ '-formyl-3-methylthiopropane-1,1-diamine (Z-gMet-For, 5g): IR ( $\mathrm{KBr}$ ): 1,658 and $1,715 \mathrm{~cm}^{1} ; \mathrm{R}_{\mathrm{f}}\left(10 \% \mathrm{MeOH} / \mathrm{CHCl}_{3}\right): 0.30$; ${ }^{1} \mathrm{H}$ NMR ( $\delta$, DMSO): 2.03 (s, 3H), $2.14(\mathrm{~m}, 2 \mathrm{H}), 2.40$ $(\mathrm{m}, 2 \mathrm{H}), 5.00(\mathrm{~s}, 2 \mathrm{H}), 5.15(\mathrm{~m}, 1 \mathrm{H}), 7.20(\mathrm{~m}, 5 \mathrm{H})$, $7.95(\mathrm{~s}, 1 \mathrm{H}), 8.09(\mathrm{~m}, 1 \mathrm{H}), 8.20(\mathrm{~m}, 1 \mathrm{H}) ;{ }^{13} \mathrm{C} \mathrm{NMR}$ ( $\delta$, DMSO): $15.26,16.20,30.21,59.64,66.90$, 127.70, 128.38, 137.30, 158.58, 163.22.

10. (S)-Methyl- $N$-benzyloxycarbonyl, $N^{\prime}$-formyl-3, 3-diaminoproanoate (Z-gAsp-(OMe)-For, 5j): IR $(\mathrm{KBr}): 1,659$ and $1,710 \mathrm{~cm}^{1} ; \mathrm{R}_{\mathrm{f}}(10 \% \mathrm{MeOH} /$ $\left.\mathrm{CHCl}_{3}\right): 0.29 ;{ }^{1} \mathrm{H}$ NMR $(\delta, \mathrm{DMSO}): 2.96(\mathrm{~m}, 2 \mathrm{H})$, $3.71(\mathrm{~s}, 3 \mathrm{H}), 5.01(\mathrm{~s}, 2 \mathrm{H}), 5.35(\mathrm{~m}, 1 \mathrm{H}), 7.23(\mathrm{~m}, 5 \mathrm{H})$, $7.91(\mathrm{~s}, 1 \mathrm{H}), 8.06(\mathrm{~m}, 1 \mathrm{H}), 8.42(\mathrm{~d}, 1 \mathrm{H}) ;{ }^{13} \mathrm{C} \mathrm{NMR}(\delta$, DMSO): 40.14, 53.44, 59.60, 65.46, 127.70, 128.36, 137.29, 156.60, 164.10, 169.72 .

11. (S)-Methyl- $N$-benzyloxycarbonyl, $\quad N^{\prime}$-formyl-4,4diaminobutanoate (Z-gGlu-(OMe)-For, 5j): IR (KBr): 1,657 and 1,709 $\mathrm{cm}^{1} ; \mathrm{R}_{\mathrm{f}}\left(10 \% \mathrm{MeOH} / \mathrm{CHCl}_{3}\right)$ : $0.28 ;{ }^{1} \mathrm{H}$ NMR $(\delta$, DMSO): $1.82(\mathrm{~m}, 2 \mathrm{H}), 2.66(\mathrm{~m}$, $2 \mathrm{H}), 3.72(\mathrm{~s}, 3 \mathrm{H}), 5.00(\mathrm{~s}, 2 \mathrm{H}), 5.32(\mathrm{~m}, 1 \mathrm{H}), 7.21(\mathrm{~m}$, $5 \mathrm{H}), 7.94(\mathrm{~s}, 1 \mathrm{H}), 8.03(\mathrm{~m}, 1 \mathrm{H}), 8.39(\mathrm{~d}, 1 \mathrm{H}) ;{ }^{13} \mathrm{C}$ NMR ( $\delta$, DMSO): 15.90, 30.10, 53.46, 59.62, 65.41, 127.72, 128.34, 137.26, 156.61, 164.12, 169.73.

12. ( $S$ )- $N$-Benzyloxycarbonyl- $N^{\prime}$-formyl-2-benzylthioethane-1,1 diamine (Z-g Cys(Bn)-For, 5k): IR (KBr): 1,659 and $1,710 \mathrm{~cm}^{1} ; \mathrm{R}_{\mathrm{f}}(10 \% \mathrm{MeOH} /$ $\left.\mathrm{CHCl}_{3}\right): 0.30 ;{ }^{1} \mathrm{H}$ NMR $(\delta$, DMSO): $3.29(\mathrm{~d}, 2 \mathrm{H})$, $3.71(\mathrm{~s}, 2 \mathrm{H}), 5.00(\mathrm{~s}, 2 \mathrm{H}), 5.49(\mathrm{~m}, 1 \mathrm{H}), 7.12-7.50$ (m, 10H), $7.95(\mathrm{~s}, 1 \mathrm{H}), 8.18(\mathrm{~m}, 1 \mathrm{H}), 8.65(\mathrm{br}, 1 \mathrm{H})$; ${ }^{13} \mathrm{C}$ NMR ( $\delta$, DMSO): $36.64,37.61,59.95,66.96$, $127.01,127.70,128.30,128.61,131.92,135.03$, 137.90, 157.70, 164.61, 175.90.

Spectral Data for Functionalized Formamides 7a-d Derived from Z-Dipeptide Acids

13. Benzyl (S)-1-[(S)-1-formamido-2-methylpropylamino]-1-oxopropan-2-ylcarbama te (Z-Ala-gValFor, 7a): IR (KBr): 1,655 and $1,705 \mathrm{~cm}^{1} ; \mathrm{R}_{\mathrm{f}}(10 \%$ $\mathrm{MeOH} / \mathrm{CHCl}_{3}$ ): $0.24 ;{ }^{1} \mathrm{H}$ NMR ( $\delta$, DMSO): 0.84 $(\mathrm{d}, 6 \mathrm{H}, \mathrm{J}=6.8 \mathrm{~Hz}), 1.20(\mathrm{~d}, 3 \mathrm{H}, \mathrm{J}=7.0 \mathrm{~Hz}), 1.99$ $(\mathrm{m}, 1 \mathrm{H}), 4.15(\mathrm{~m}, 1 \mathrm{H}), 4.98(\mathrm{~s}, 2 \mathrm{H}), 5.28(\mathrm{~m}, 1 \mathrm{H}), 7.28$ $(\mathrm{m}, 5 \mathrm{H}), 7.96(\mathrm{~s}, 1 \mathrm{H}), 8.15(\mathrm{~m}, 1 \mathrm{H}), 8.41(\mathrm{~m}, 1 \mathrm{H})$; ${ }^{13} \mathrm{C}$ NMR ( $\delta$, DMSO): 18.09, 20.22, 31.80, 54.40, $61.67,66.15,127.21,127.79,136.38,155.61,163.66$, 172.92 . 
14. Benzyl (S)-2-[(S)-1-formamidoethylamino]-2-oxo1-phenylethylcarbamate (Z-L-Phg-gAla-For, 7b): IR (KBr): 1,662 and $1,712 \mathrm{~cm}^{1} ; \mathrm{R}_{\mathrm{f}}(10 \% \mathrm{MeOH} /$ $\left.\mathrm{CHCl}_{3}\right): 0.23 ;{ }^{1} \mathrm{H} \mathrm{NMR}(\delta, \mathrm{DMSO}): 1.26(\mathrm{~d}, 3 \mathrm{H}$, $\mathrm{J}=7.6 \mathrm{~Hz}), 4.61(\mathrm{~m}, 1 \mathrm{H}), 5.00(\mathrm{~s}, 1 \mathrm{H}), 5.21(\mathrm{~m}$, $1 \mathrm{H}), 7.27-7.43(\mathrm{~m}, 10 \mathrm{H}), 7.94(\mathrm{~s}, 1 \mathrm{H}), 8.04(\mathrm{~m}, 1 \mathrm{H})$, $8.18(\mathrm{~m}, 1 \mathrm{H}) ;{ }^{13} \mathrm{C}$ NMR $(\delta, \mathrm{DMSO}): 20.64,54.44$, 57.80, 66.12, 126.34, 127.20, 127.75, 127.81, 130.90, 133.27, 136.20, 155.61, 164.10, 172.

15. Benzyl (S)-1-[(S)-1-formamido-2-methylpropylamino]-4-methyl-1-oxopentan-2-ylcarbamate (ZLeu-gVal-For, 7f): IR (KBr): 1,659 and $1,710 \mathrm{~cm}^{1}$; $\mathrm{R}_{\mathrm{f}}\left(10 \% \mathrm{MeOH} / \mathrm{CHCl}_{3}\right): 0.23 ;{ }^{1} \mathrm{H} \mathrm{NMR}(\delta, \mathrm{DMSO})$ : $0.90(\mathrm{~m}, 12 \mathrm{H}), 1.51-2.30(\mathrm{~m}, 4 \mathrm{H}), 3.88(\mathrm{~m}, 1 \mathrm{H}), 4.95$ (s, 2H), $5.25(\mathrm{~m}, 1 \mathrm{H}), 7.27(\mathrm{~m}, 5 \mathrm{H}), 7.94(\mathrm{~s}, 1 \mathrm{H}), 8.08$ $(\mathrm{m}, 1 \mathrm{H}), 8.18(\mathrm{~m}, 1 \mathrm{H}) ;{ }^{13} \mathrm{C} \mathrm{NMR}(\delta, \mathrm{DMSO}): 18.44$, 25.30, 31.71, 35.60, 51.44, 57.780 127.20, 128.81, 136.36, 156.30, 164.10, 172.29.

16. Benzyl 2-\{[(S)-1-formamido-2-methylpropyl $]$ carbamoyl\}pyrrolidine-1-carboxyla te (Z-Pro-gValFor, 7d): IR (KBr): 1,659 and $1,710 \mathrm{~cm}^{1} ; \mathrm{R}_{\mathrm{f}}(10 \%$ $\mathrm{MeOH} / \mathrm{CHCl}_{3}$ ): $0.24 ;{ }^{1} \mathrm{H} \mathrm{NMR}(\delta, \mathrm{DMSO}): 0.73$ (d, 6H, J = 7.4 Hz), 1.77 (br, 4H), $1.86(\mathrm{~m}, 1 \mathrm{H}), 3.34$ $(\mathrm{m}, 2 \mathrm{H}), 4.18(\mathrm{~m}, 1 \mathrm{H}), 4.96(\mathrm{~s}, 2 \mathrm{H}), 5.20(\mathrm{~m}, 1 \mathrm{H})$, $7.27(\mathrm{~m}, 5 \mathrm{H}), 7.95(\mathrm{~s}, 1 \mathrm{H}), 8.05(\mathrm{~m}, 1 \mathrm{H}), 8.32(\mathrm{~m}$, $1 \mathrm{H}) ;{ }^{13} \mathrm{C}$ NMR $(\delta, \mathrm{DMSO}): 18.45,23.80,28.71$, $31.71,46.90,54.44,61.19,67.15,127.80,128.88$, $128.45,136.99,155.45,164.20,173.21$.

\section{Results and Discussions}

In a typical reaction $\mathrm{Z}-\mathrm{Ala}-\mathrm{OH}, \mathbf{1 b}$ was converted into its corresponding acid azide on treatment of its mixed anhydride $\mathbf{2 b}$ with sodium azide. The azide $\mathbf{3}$ was subjected to Curtius rearrangement following any one of the methods: thermal (Chorev et al. 1977; Patil et al. 2003) or irradiation using microwaves or subjecting to ultrasonication (Sureshbabu et al. 2005) to accomplish the conversion in to isocyanate $\mathbf{4 b}$. Complete conversion into isocyanate was confirmed by the disappearance of the azide peak at $2,110 \mathrm{~cm}^{-1}$ and the appearance of a distinct isocyanate peak at $2,230 \mathrm{~cm}^{-1}$ in IR analysis. The dichloromethane solution of $\mathbf{4 b}$ was then treated with $96 \%$ formic acid in presence of catalytic amount of DMAP at $0^{\circ} \mathrm{C}$ and the reaction was continued until completion by monitoring through TLC. The desired functionalized formamide $\mathbf{5 b}$ conveniently precipitated from the reaction mixture. The addition of DMAP to the isocyanate solution was done in the view of the known activation of isocyanate towards the attack by the carbonyl oxygen upon complexation with DMAP (Schuemacher and Hoffmann 2001). Further the requirement of DMAP to drive the reaction was evident when the uncatalyzed reaction showed very less formation of the product even with higher equivalents of formic acid. As a catalyst, DMAP was meritorious over the other tertiary amines like $N$-methylmorpholine (NMM), triethyl amine (TEA) and pyridine as these resulted in lesser yields even with higher equivalents. Increase of temperature to 25 ${ }^{\circ} \mathrm{C}$ also resulted in lesser yields due to the formation of mixture of impurities. Employing this protocol (Scheme 1), a variety of isocyanates derived from Z-amino acids was converted to related formamides $\mathbf{5 a - 1}$ on a 10 mmole scale (Table 1). All the compounds made were obtained in good yield and fully characterized using ${ }^{1} \mathrm{H}$ NMR, ${ }^{13} \mathrm{C}$ NMR and mass spectrometry.

The protocol was extended to the synthesis of formamides derived from $\mathrm{Z}$-protected peptide acids. The peptide acids were synthesized by coupling with $O, N$-bis-trimethylsilyl amino acid with mixed anhydride of Z-amino acids (Tantry and Sureshbabu 2004). The dipeptide acids were further converted to corresponding isocyanates through the

Table $1 N$-Benzyloxycarbonyl-N'-formyl-gem-diaminoalkyl derivatives 5a-k prepared through Scheme 1

\begin{tabular}{|c|c|c|c|c|c|}
\hline Compound & $\mathrm{R}_{1}$ & Yield\% & M.p ${ }^{\circ} \mathrm{C}$ & HRMS $[\mathrm{M}+\mathrm{Na}]^{+}$Calc. & HRMS $[\mathrm{M}+\mathrm{Na}]^{+}$Observed \\
\hline $5 \mathbf{a}$ & $\mathrm{H}$ & 90 & $162-63$ & 231.0746 & 231.0750 \\
\hline $5 \mathbf{b}$ & $\mathrm{CH}_{3}$ & 91 & $132-33$ & 245.0902 & 245.0906 \\
\hline $5 c$ & $\mathrm{CH}_{2} \mathrm{C}_{6} \mathrm{H}_{5}$ & 92 & $174-75$ & 321.1215 & 321.1213 \\
\hline $5 d$ & $\mathrm{CH}\left(\mathrm{CH}_{3}\right)_{2}$ & 90 & $138-39$ & 273.1215 & 273.1218 \\
\hline $5 e$ & $\mathrm{CH}_{2} \mathrm{CH}\left(\mathrm{CH}_{3}\right)_{2}$ & 85 & $123-24$ & 287.1372 & 287.1368 \\
\hline $5 f$ & $\mathrm{CH}\left(\mathrm{CH}_{3}\right) \mathrm{CH}_{2} \mathrm{CH}_{3}$ & 86 & $148-49$ & 287.1372 & 287.1371 \\
\hline $5 g$ & $\mathrm{C}_{6} \mathrm{H}_{5}(\mathrm{~L})$ & 90 & $158-59$ & 307.1059 & 307.1057 \\
\hline $5 \mathbf{h}$ & $\mathrm{C}_{6} \mathrm{H}_{5}$ (D) & 91 & $160-61$ & 307.1059 & 307.1061 \\
\hline $5 i$ & $\left(\mathrm{CH}_{2}\right)_{2} \mathrm{SCH}_{3}$ & 84 & $150-51$ & 305.0936 & 305.0933 \\
\hline $5 \mathbf{j}$ & $\mathrm{CH}_{2} \mathrm{COOCH}_{2} \mathrm{C}_{6} \mathrm{H}_{5}$ & 80 & $108-09$ & 379.1270 & 379.1274 \\
\hline $5 \mathbf{k}$ & $\left(\mathrm{CH}_{2}\right)_{2} \mathrm{COOCH}_{2} \mathrm{C}_{6} \mathrm{H}_{5}$ & 78 & $102-03$ & 393.1426 & 393.1423 \\
\hline 51 & $\mathrm{CH}_{2} \mathrm{SCH}_{2} \mathrm{C}_{6} \mathrm{H}_{5}$ & 86 & $140-41$ & 367.1092 & 367.1094 \\
\hline
\end{tabular}


Scheme 2 Synthesis of $\mathrm{N}$-formylated gem-diaminoalkyl derivatives of Z-dipeptides<smiles>[R]C(NC(=O)C([R])NC(=O)OCc1ccccc1)C(=O)O</smiles>

6a-g

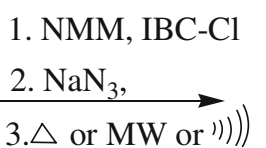

4. $\mathrm{HCOOH} / \mathrm{DMAP}$<smiles>[R]C([R])NC([R])NC(=O)NC([R])C(=O)NC(=O)OCc1ccccc1</smiles>

7a-g

Table $2 N$-formylated gem-diaminoalkyl derivatives of dipeptides prepared through Scheme 2

\begin{tabular}{lllllll}
\hline Compound & $\mathrm{R}_{1}$ & $\mathrm{R}_{2}$ & Yield \% & M.p ${ }^{\circ} \mathrm{C}$ & HRMS [M+Na $]^{+} \mathrm{Calc}$. & HRMS [M+Na] ${ }^{+} \mathrm{Observed}$ \\
\hline $\mathbf{7 a}$ & $\mathrm{CH}_{3}$ & $\mathrm{CH}\left(\mathrm{CH}_{3}\right)_{2}$ & 75 & $150-51$ & 344.1586 & 344.1589 \\
7b & $\mathrm{C}_{6} \mathrm{H}_{5}(\mathrm{~L})$ & $\mathrm{CH}_{3}$ & 78 & $161-62$ & 378.1430 & 378.1426 \\
7c & $\mathrm{C}_{6} \mathrm{H}_{5}(\mathrm{D})$ & $\mathrm{CH}_{3}$ & 79 & $164-65$ & 378.1430 & 378.1428 \\
$\mathbf{7 d}$ & $-\left(\mathrm{CH}_{2}\right)_{3}-$ & $\mathrm{CH}\left(\mathrm{CH}_{3}\right)_{2}$ & 73 & $141-44$ & 370.1743 & 370.1739 \\
$\mathbf{7 e}$ & $\mathrm{CH}_{2} \mathrm{C}_{6} \mathrm{H}_{5}$ & $\mathrm{CH}_{3}$ & 81 & $158-60$ & 392.1586 & 392.1590 \\
7f & $\mathrm{CH}_{2} \mathrm{CH}\left(\mathrm{CH}_{3}\right)_{2}$ & $\mathrm{CH}\left(\mathrm{CH}_{3}\right)_{2}$ & 70 & $133-34$ & 386.2056 & 386.2053 \\
\hline
\end{tabular}

rearrangements of parent acid azides under reflux or microwave irradiation and reacted with $\mathrm{HCOOH}$ in presence of DMAP to obtain the required formamides $7 \mathbf{a}-\mathbf{f}$ in good yields (Scheme 2, Table 2).

Epimerization during the course of the reaction was examined by recording the ${ }^{1} \mathrm{H}$ NMR spectrum of crude $\mathbf{7 b}$ and $\mathbf{7 c}$ synthesized using the present method. $\mathbf{7 b}$ contained a doublet at 1.29, 1.31 corresponding to the methyl group protons. While its other epimer, $7 \mathbf{c}$ had the $-\mathrm{CH}_{3}$ doublet at $1.21,1.22$. Furthermore, the equimolar mixture containing 7b and 7c had peaks at four different $\delta$ values, 1.21, 1.25, $1.29,1.32$. This demonstrated that the proposed procedure was epimerization free.

\section{Conclusions}

We have demonstrated that the isocyanate derived from Z-amino acid/peptide acid azides can be used as an easily accessible functionality to generate various $N$-benzyloxycarbonyl $N^{\prime}$-formyl gem-diaminoalkyl compounds. The synthesis involves Curtius rearrangement of $\mathrm{Z}$ - $\alpha$-amino acyl azides into related isocyanate and reaction of the latter with formic acid under DMAP catalysis. The procedure is operationally simple, high yielding and enables formylation of the isocyanates under mild conditions.

Acknowledgements We are grateful to the Council of Scientific \& Industrial Research, Govt. of India for financial support and S.I.F and M.B.U of I.I.Sc., Bangalore for providing NMR and mass facilities.

\section{References}

Berman JM, Goodman M (1984) Synthesis of cyclic and acyclic partially modified retro-inverso modified enkaphalins. Int J Pept Prot Res 23:610-620
Chorev M (2005) The partial retro-inverso modification: A road traveled together. Biopolymers (Pept Sci) 80:67-84

Chorev M, Goodman M (1983) Partially modified retro-inverso peptides. Int J Pept Prot Res 21:258-268

Chorev M, Willson CG, Goodman M (1977) A general approach to retro-isomeric linear peptide synthesis. J Am Chem Soc 99: 8075-8076

Chorev M, MacDonald SA, Goodman M (1984) Retro-inverso isomerization of peptides: side reactions in the synthesis of N,N'-diacyl-1,1-diamino-2-phenylethane derivatives. J Org Chem 49:821-827

Domling A, Ugi I (2000) Multicomponent reactions with isocyanides. Angew Chem Int Edit 39:3168-3210

Fischer L, Semetey V, Lozano J-M, Schaffner A-P, Briand J-P, Didierjean C, Guichard G, (2007) Succinimidyl carbamate derivatives from $N$-Protected $\alpha$-amino acids and dipeptidesSynthesis of ureidopeptides and oligourea/peptide hybrids. Eur J Org Chem 2511-2525

Fletcher MD, Campbell MM (1998) Partially modified retro-inverso peptides: development, synthesis, and conformational behavior. Chem Rev 98:763-796

Fuller WP, Goodman M, Verlander MS (1985) A new class of amino acid based sweeteners. J Am Chem Soc 107:5821-5822

Loudon GM, Almond MR, Jacob JN (1981) Mechanism of hydrolysis of N-(1-aminoalkyl)amides. J Am Chem Soc 103:4508-4515

Pallai PV, Richman S, Struthers RS, Goodman M (1983) Approaches to the synthesis of retro-inverso peptides. Int J Pept Protein Res 21:84-92

Parham ME, Louden GM (1978) Carboxyl-terminal sequential degradation of peptides. Biochem Biophys Res Commun 80:1-6

Patil BS, Vasanthakumar GR, Sureshbabu VV (2003) Isocyanates of $\mathrm{N} \alpha$-[(9-Fluorenylmethyl)oxy]carbonyl amino acids: synthesis, isolation, characterization, and application to the efficient synthesis of urea peptidomimetics. J Org Chem 68:7274-7280

Sarvari MH, Sharghi $\mathrm{H}$ (2006) $\mathrm{ZnO}$ as a new catalyst for $\mathrm{N}$ formylation of amines under solvent-free conditions. J Org Chem 71:6652-6654 and reference cited therein

Scheibler L, Chorev M (2003) Synthesis of retroinverso-peptides. In: Goodman M, Felix A, Moroder L, Toniolo C (eds) Methods of organic chemistry (Houben-Weyl): synthesis of peptides and peptidomimetics, vol. E22c. Georg Thieme Verlag Stuttgart, New York, pp 528-551

Schuemacher AC, Hoffmann RW (2001) Condensation between isocyanates and carboxylic acids in the presence of 
4-Dimethylaminopyridine (DMAP), a mild and efficient synthesis of amides. Synthesis 243-246

Sudarshan NS, Narendra N, Hemantha HP, Sureshbabu VV (2007) An efficient conversion of the carboxylic group of $N$-fmoc $\alpha$-amino acids/peptide acids into $N$-formamides employing isocyanates as key intermediates. J Org Chem 72:9804-9807

Sureshbabu VV, Kantharaju, Tantry SJ (2005) Curtius rearrangement using ultrasonication: isolation of isocyanates of Fmoc-amino acids and their utility for the synthesis of dipeptidyl ureas. Int $\mathbf{J}$ Pept Res Therapeutics 11:131-137

Sureshbabu VV, Patil BS, Venkataramanarao R (2006) Preparation, isolation, and characterization of $\mathrm{N} \alpha$-Fmoc-peptide isocyanates: solution synthesis of oligo- $\alpha$-peptidyl ureas. J Org Chem 71:7697-7705
Tantry SJ, Sureshbabu VV (2004) Synthesis of N $\alpha$-protected peptide acids by the $\mathrm{N} \rightarrow \mathrm{C}$ chain extension employing $\mathrm{O}, \mathrm{N}$-bistrimethylsilyl amino acids using the mixed anhydride method. Indian J Chem 43B:1282-1287

Venkataramanarao R, Sureshbabu VV (2006) Synthesis of retroinverso peptides employing isocyanates of $\mathrm{N} \alpha$-Fmoc-amino acids/peptide acids catalyzed by DMAP. Tetrahedron Lett 47:9139-9141

Venkataramanarao R, Sudarshan NS, Sureshbabu VV (2007) Microwave assisted alcoholysis of isocyanates derived from $\mathrm{N} \alpha-[(9-$ fluorenylmethyl)oxy]carbonyl amino acids: synthesis of $\mathrm{N}$ Fmoc-N1-Z-/Boc-/Alloc-/Bsmoc-gem-diamines. Int J Pept Res Therapeutics 13:393-397 